\title{
A General Equilibrium Model of the Term Structure of Interest Rates under Regime-switching Risk
}

\author{
Shu Wu and Yong Zeng*
}

First draft: March 2003

This version: December 2003

\begin{abstract}
This paper incorporates the systematic risk of regime shifts into a general equilibrium model of the term structure of interest rates. The model shows that there is a new source of time-variation in bond term premiums in the presence of regime shifts. This new component is a regime-switching risk premium that depends on the covariations between discreet changes in marginal utility and bond prices across different regimes. A closed-form solution for the term structure of interest rates is obtained under an affine model using log-linear approximation. The model is estimated by Efficient Method of Moments. The regime-switching risk is found to be statistically significant and mostly affect the long-end of the yield curve.
\end{abstract}

JEL Classification: G12, E43

Key Words: The Term Structure, General Equilibrium, Markov Regime Shifts

* Shu Wu is with Department of Economics at The University of Kansas, Summerfield Hall 213, Lawrence, KS 66045. Tel: (785) 864 2868, email: shuwu@ku.edu. Yong Zeng is with Department of Mathematics and Statistics at University of Missouri at Kansas City, Kansas City, MO 64110. Tel: (816) 235 5850, email: zeng@mendota.umkc.edu. We are grateful to Ming Liu, Cyrus Ramezani and Ken Singleton for comments and suggestions. We also thank participants at 2003 Missouri Economics Conference, 2003 AMS-IMS-SIAM Summer Research Conference on Mathematics of Finance, and at seminars of University of Wisconsin at Madison and The University of Kansas for helpful comments. Ikuyasu Usui provided able research assistance. 


\section{Introduction}

There exists a large body of empirical evidence suggesting that the aggregate economy is characterized by periodic shifts between distinct regimes of the business cycle (e.g. Hamilton 1989, Filardo 1994 and Diebold and Rudebusch 1996). A number of researchers have successfully used Markov regime-switching models to fit the dynamics of the short-term interest rate (see, among others, Hamilton 1988, Garcia and Perron 1996, Gary 1996 and Ang and Bakeart 1998). ${ }^{1}$ These results have motivated the recent studies of the impact of regime shifts on the entire yield curve using dynamic term structure models. A common approach, as in Naik and Lee (1997), Boudoukh et al.(1999), Evans (2001) and Bansal and Zhou (2002), is to incorporate Markov-switching into the stochastic processes of the pricing kernel and/or state variables. ${ }^{2}$ The regime-dependence introduced by these studies implies richer dynamic behavior of the market price of risk and therefore offers greater econometric flexibility for the term structure models to simultaneously account for the time series and cross-sectional properties of interest rates. However, as pointed out by Dai and Singleton (2003), the risk of regime shifts is not priced in these models, and hence it does not contribute independently to bond risk premiums.

Without pricing the risk of regime shifts, the previous studies have essentially treated the regime shifts as an idiosyncratic risk that can be diversified away by bond investors. However, most of these studies (see, for example, Bansal and Zhou, 2002.) have also shown that regimes are intimately related to the business cycle, suggesting a close link between the regime shift and aggregate uncertainties. ${ }^{3}$ Hence it is most likely that the Markov regime shifts represent a systematic risk which should be priced in the term structure models.

The main objective of current paper is to extend this strand of literature by developing a dynamic term structure model under the systematic risk of regime shifts in a general equilibrium setting similar to Cox, Ingersoll and Ross (1985a, 1985b). Our model implies that bond risk premiums include two components under regime shifts. One is a regime-dependent risk premium due to diffusion risk as in the previous studies. This risk premium has added econometric flexibilities, as demonstrated in the previous empirical studies, relative to those in single-regime models because of the Markov-shift of the underlying parameters. The other is a regime-switching risk premium that depends on the covariations between the discreet changes in marginal utility and bond prices across different regimes. Therefore the model shows that there is an additional source of time-variation in bond risk premiums in the presence of regime shifts. This new component of the term premiums is associated with the systematic risk of periodic shifts in bond prices (or interest rates) due to regime changes.

\footnotetext{
${ }^{1}$ The expectation theory is usually invoked to relate long-term interest rates to the short rate in this literature, such as in Ang and Bakeart (1998).

${ }^{2}$ Other studies of the term structure of interest rates under hidden Markov chains include Bielecki and Rutkowski (2001), Elliott and Mamon (2001) among others.

${ }^{3}$ Following Bansal and Zhou (2002), we plot in Figure 2 the implied regimes by the term structure model in Section 3 below and the business cycle expansions and recessions identified by NBER. The figure clearly shows that the regimes are related to the fluctuations of the aggregate economy.
} 
Given the empirical evidence from the previous studies that the yield curve exhibits significantly different properties across regimes, the model implies that the regime-switching risk is likely to be an important factor that affects bond returns.

We further obtain a closed-form solution of the term structure of interest rates under an affine-type model using the log-linear approximation similar to that in Bansal and Zhou (2002). The model is estimated by Efficient Method of Moments (EMM) developed in Bansal et al. (1995) and Gallant and Tauchen (1996, 2001). We use the monthly data on 6-month treasury bill and 5-year treasury bond from 1964 to 2000 in the estimation. We find that the market price of regime-switching risk is highly significant and affects mostly the long-end of the yield curve. The regime-switching risk, as expected, accounts for a significant portion of the term premiums for long-term bond.

In a closely related study, Dai, Singleton and Yang (2003) develop and estimate a multifactor dynamic term structure model with regime-shift risk based on no-arbitrage condition. Their results also strongly reject the null hypothesis that the regime-shift risk is not priced in the U.S. treasury bond yields. They show that the model with priced regime-switching risk can capture some salient features of term structure of interest rates. The focus of this paper is to derive the term structure model from a general equilibrium setting that allows us to further elucidates the economic nature of the regime-switching risk, which is the main difference between the current paper and Dai et al. (2003). Another difference is our model is specified in continuous time and the model allows the factor loadings in the term structure to be regime-dependent, while the model in Dai et al. (2003) is in discreet time and the regime shifts have no impact on the factor loadings. ${ }^{4}$

The rest of the paper is organized as follows. Section 2 specifies the underlying economy and lays out the asset pricing model under the systematic regime-switching risk. Section 3 obtains the closed-form solution for the term structure of interest rates. Section 4 discusses the empirical results from EMM estimation and section 5 concludes.

\section{The Model}

\subsection{The Underlying Economy}

Consider an economy with a single good and a large number of infinitely lived and identical agents similarly to that in Cox, Ingersoll and Ross (1985a, 1985b) (henceforth CIR). We first describe the state variables and investment opportunities for the economy as well as the representative agent's objective function below.

\footnotetext{
${ }^{4}$ The latter feature of the model in Dai et al. (2003) allow them to obtain the exact solution to the term structure of interest rates without resorting to the log-linear approximation. Their model also allows the regime-switching probabilities to be state-dependent. While the transition intensities across regimes are assumed to be constant in the estimated term structure model of the current paper, our main theoretical results do not depend on this assumption.
} 


\subsubsection{State Variables}

We assume that the economy is driven by two state variables. ${ }^{5}$ One of the state variable $x(t)$ has a continuous path and is determined by the stochastic differential equation

$$
d x=\mu_{x} d t+\sigma_{x} d B_{t}
$$

where the drift term $\mu_{x}$ and the diffusion term $\sigma_{x}$ are in general time-varying and regimedependent. The other state variable is a continuous-time Markov chain $s(t)$ taking on values of $1,2, \cdots, N$ if there are $N$ distinct regimes. Following Landen (2000), we make use of the marked point process to obtain a convenient representation of $s(t)$. In particular, the mark space $E$, which contains all possible regime shifts, is defined as

$$
E=\{(i, j): i \in\{1, \ldots, N\}, j \in\{1, \ldots, N\}, i \neq j\}
$$

with $\sigma$-algebra $\mathcal{E}=2^{E}$. Denote $z=(i, j)$ as a generic point in $E$, representing a regime shift from state $i$ to $j$. A marked point process, $m(t, \cdot)$ is uniquely characterized by its stochastic intensity kernel, ${ }^{6}$, which can be defined as

$$
\gamma_{m}(d t, d z)=h(z, x(t-)) \mathbf{I}\{s(t-)=i\} \epsilon_{z}(d z) d t,
$$

where $h(z, x(t-))$ is the regime-shift (from regime $i$ to $j$ ) intensity at $z=(i, j)$ conditional on $x(t-), \mathbf{I}\{s(t-)=i\}$ is an indicator function of regime at time $t-$, and $\epsilon_{z}(A)$ is the Dirac measure for $A$, a subset of $E$, at point $z$ (defined by $\epsilon_{z}(A)=1$ if $z \in A$ and 0 , otherwise). Heuristically, for $z=(i, j), \gamma_{m}(d t, d z)$ can be thought of as the conditional probability of shifting from Regime $i$ to Regime $j$ during $[t, t+d t)$ given $x(t-)$ and $s(t-)=i$. Note that $\gamma_{m}(d t, d z)$ is in general state dependent.

Let $A$ be a subset of $E$. Then $m(t, A)$ counts the cumulative number of regime shifts that belong to $A$ during $(0, t] . m(t, A)$ has its compensator, $\gamma_{m}(t, A)$, given by

$$
\gamma_{m}(t, A)=\int_{0}^{t} \int_{A} h(z, x(\tau-)) \mathbf{I}\{s(\tau-)=i\} \epsilon_{z}(d z) d \tau .
$$

This simply implies that $m(t, A)-\gamma_{m}(t, A)$ is a martingale.

Using the above notations, the evolution of the regime $s(t)$ can be conveniently represented as

$$
d s=\int_{E} \zeta(z) m(d t, d z)
$$

with the compensator given by

$$
\gamma_{s}(t) d t=\int_{E} \zeta(z) \gamma_{m}(d t, d z), \text { where } \zeta(z)=\zeta((i, j))=j-i
$$

\footnotetext{
${ }^{5}$ It is straight forward to extend the model to include more state variables. We keep the model as simple as possible to ease exposition.

${ }^{6}$ See Last and Brandt (1995) for detailed discussion of marked point process, stochastic intensity kernel and related results.
} 
For example, if there is a regime shift from $i$ to $j$ occurrs at time $t$, equation (4) then implies $s_{t}=(j-i)+s_{t-}=j$. Note that $\int_{E}$ is equivalent to the double summation $\sum_{j \neq i} \sum_{i}$.

\subsubsection{Investment Opportunities}

Without loss of generality, we assume that the output is produced by a single technology which depends on both state variables $x(t)$ and $s(t)$ as described by the following stochastic differential equation

$$
d y=y \mu_{y} d t+y \sigma_{y} d B_{t}+\int_{E} y \delta_{y}(z) m(d t, d z)
$$

where both $\mu_{y}$ and $\sigma_{y}$ can be functions of $x(t-)$ and $s(t-)$. And $\delta_{y}(z)$ is the discrete percentage change in $y$ due to a regime shift, i.e. $\delta_{y}(z)=\frac{y(t, s(t))-y(t-s(t-))}{y(t-s(t-))}$. Therefore we assume that regime shifts not only affect the drift $\mu_{y}$ and the volatility $\sigma_{y}$, they also directly result in discontinuous changes in the output process as the economy shifts from one regime to another.

As in CIR model, we assume that there is a competitive market for instantaneous borrowing and lending at the short-term interest rate $r(t)$. There is also a competitive market for default-free pure discount bonds whose prices are given by

$$
d F=F \mu_{F} d t+F \sigma_{F} d B_{t}+\int_{E} F \delta_{F}(z) m(d t, d z)
$$

where $\mu_{F}, \sigma_{F}$ and $\delta_{F}(z)$ are to be determined by the equilibrium conditions. And $\delta_{F}(z)$ is the discrete percentage change in the bond prices due to a regime shift, i.e. $\delta_{F}(z)=$ $\frac{F(t, s(t))-F(t-, s(t-))}{F(t-, s(t-))}$. Hence, unlike the conventional models, we allow regime shifts not only affect the conditional distribution of bond prices $\left(\mu_{F}, \sigma_{F}\right)$, but also lead to discontinuous jumps in the prices when regime shifts occur.

Note that $(7)$ can be alternatively written as

$$
\begin{aligned}
d F=F \mu_{F} d t & +\int_{E} F \delta_{F}(z) \gamma_{m}(d t, d z) \\
& +F \sigma_{F} d B_{t}+\int_{E} F \delta_{F}(z)\left[m(d t, d z)-\gamma_{m}(d t, d z)\right]
\end{aligned}
$$

The last two terms in the above equation are martingales. Therefore (8) implies that the instantaneous expected bond return is

$$
E_{t-}\left(\frac{d F}{F}\right)=\mu_{F} d t+\int_{E} \delta_{F}(z) \gamma_{m}(d t, d z)
$$

where the first term is the regime-dependent expected bond return due to diffusion, and the last term is an additional component in the bond return due to discrete regime shifts. 


\subsubsection{The Representative Agent's Objective Function}

Given the initial wealth $w_{0}$, a representative agent seeks to maximize her expected lifetime utility given by

$$
E_{0}\left[\int_{0}^{\infty} e^{-\rho t} U(c(t)) d t\right]
$$

where $c(t)$ is the flow of consumption and $U(\cdot)$ is the instantaneous utility function. As usual, $U(\cdot)$ is assumed to be strictly concave, increasing and twice differentiable with $U(0)=0$ and $U^{\prime}(0)=\infty$.

At each instant, the representative agent allocates her wealth among investment in the physical production, the discount bonds, the risk-free borrowing and lending and consumption. We assume that both physical investment and trading of the financial assets take place in continuous time without borrowing constraint, transaction cost and all other forms of market friction. The agent's budget constraint is therefore given by

$$
d w=w \mu_{w} d t+w \sigma_{w} d B_{t}+\int_{E} w \delta_{w}(z) m(d t, d z)
$$

where

$$
\begin{array}{r}
w \mu_{w}=w\left[\phi_{1}\left(\mu_{y}-r\right)+\phi_{2}\left(\mu_{F}-r\right)+r\right]-c \\
w \sigma_{w}=w\left[\phi_{1} \sigma_{y}+\phi_{2} \sigma_{F}\right] \\
w \delta_{w}(z)=w\left[\phi_{1} \delta_{y}(z)+\phi_{2} \delta_{F}(z)\right]
\end{array}
$$

In the above equations, $w(t)$ is the agent's wealth at time $t, \phi_{1}$ is the proportion of her wealth invested in the physical production, $\phi_{2}$ is the proportion of her wealth invested in the discount bonds, and $c(t)$ is her flow of consumption. As we can clearly see from these equations, regime shifts have two effects on the investor's wealth process. On one hand, they affect the the drift and diffusion term of the investor's wealth (both $\mu_{w}$ and $\sigma_{w}$ are regime-dependent). On the other hand, they also create discontinuous changes in the wealth process when the economy shifts between distinct regimes. This is a more general setup than the conventional models where regime shifts are assumed to only have the first effect on the investor's wealth.

\subsection{The Equilibrium Bond Returns}

In this section we state the main asset pricing results for the economy subject to the systematic regime-switching risk. The detailed derivations are provided in the appendices.

Let $J(w(t), s(t), x(t))=\sup _{\left\{c, \phi_{1}, \phi_{2}\right\}} E_{t}\left[\int_{t}^{\infty} e^{-\rho(\tau-t)} U(c(\tau)) d \tau\right]$, be the indirect utility function. We use the following notations in our discussions below: $J_{w}=\frac{\partial J}{\partial w}, J_{x}=\frac{\partial J}{\partial x}$, 
$J_{w w}=\frac{\partial^{2} J}{\partial w^{2}}$ and $J_{w x}=\frac{\partial^{2} J}{\partial w \partial x}$. We also use $w^{c}$ to represent the continuous part of the wealth process. As in CIR, we denote $\operatorname{Var}\left(w^{c}\right)=\left(w \sigma_{w}\right)^{2}, \operatorname{Var}(x)=\sigma_{x}^{2}$, and $\operatorname{Cov}\left(w^{c}, x\right)=$ $\left(w \sigma_{w}\right) \sigma_{x}$. To further simplify notations, we define $\Delta_{s} f=f(s(t))-f(s(t-))$ for any function $f(\cdot)$ that depends on $s(t) . \Delta_{s} f$ is therefore the difference in $f(\cdot)$ due to a regime shift at time $t$.

The following two propositions give the equilibrium instantaneous short-term interest rate and the expected excess rate of return on a bond respectively.

Proposition 1 The equilibrium short-term interest rate is given by

$$
\begin{aligned}
r=\mu_{y}^{*}-\left(-\frac{J_{w w}}{J_{w}}\right) \frac{\operatorname{Var}\left(w^{c}\right)}{w} & -\left(-\frac{J_{w x}}{J_{x}}\right) \frac{\operatorname{Cov}\left(w^{c}, x\right)}{w} \\
& -\int_{E}\left(-\frac{\Delta_{s} J_{w}}{J_{w}}\right) \frac{\Delta_{s} w}{w} \gamma_{m}(d z)
\end{aligned}
$$

where

$$
\mu_{y}^{*}=\mu_{y}+\int_{E} \delta_{y}(z) \gamma_{m}(d z)
$$

and

$$
\gamma_{m}(d z)=h(z, x(t-)) \mathbf{I}\{s(t-)=i\} \epsilon_{z}(d z)
$$

Note that $\mu_{y}^{*} d t$ is the instantaneous expected rate of return on the production technology $E_{t-}\left(\frac{d y(t)}{y(t-)}\right)$. And $\frac{\Delta_{s} w}{w}$ and $\frac{\Delta_{s} J_{w}}{J_{w}}$ in (15) are the discrete percentage changes in $w$ and $J_{w}$ respectively due to a regime shift, i.e. $\frac{\Delta_{s} w}{w}=\delta_{w}(z)=\phi_{1} \delta_{y}(z)+\phi_{2} \delta_{F}(z)$, and

$$
\frac{\Delta_{s} J_{w}}{J_{w}}=\frac{J_{w}\left(w\left(1+\delta_{w}(z)\right), s+\zeta(z), x\right)-J_{w}(w, s, x)}{J_{w}(w, s, x)}
$$

Proposition 1 implies that the instantaneous short-term interest rate $r(t)$ is a function of both state variables $x(t)$ and $s(t)$ because $\mu_{y}^{*}, \operatorname{Var}\left(w^{c}\right), \operatorname{Cov}\left(w^{c}, x\right)$ and the marginal utility $J_{w}$ are regime-dependent. If the regime shifts is not a systematic risk as assumed in the previous literature, $\frac{\Delta_{s} J_{w}}{J_{w}}$ would be equal to zero. Otherwise the short-term interest rate will also be affected by this term. Since the indirect utility function $J(w, s, x)$ is concave in $w$, if it is also separable in $w$ and $s$ (as in the case of log utility function), we will have

$$
\left(-\frac{\Delta_{s} J_{w}}{J_{w}}\right) \frac{\Delta_{s} w}{w}>0
$$

Therefore the systematic regime-switching risk tends to lower the equilibrium short-term interest rate, as the local risk-free borrowing and lending opportunity offers a hedge against such risk. This result is similar to the impact of a systematic jump risk on the interest rate as shown in Ahn and Thompson (1988). Nevertheless, $\left(-\frac{\Delta_{s} J_{w}}{J_{w}}\right) \frac{\Delta_{s} w}{w}$ is not always positive 
in general, because $J_{w}$ also depends on $s$. Therefore the regime-switching risk has a different effect than that of jump risk even though they both lead to discontinuous changes in the marginal utility and wealth.

Proposition 2 Let $\mu_{F}^{*} d t=E_{t-}\left(\frac{d F(t)}{F(t-)}\right)$, be the instantaneous expected rate of return of a discount bond. In equilibrium, we have

$$
\begin{aligned}
\mu_{F}^{*}-r & =\left[\left(-\frac{J_{w w}}{J_{w}}\right) \operatorname{Var}\left(w^{c}\right)+\left(-\frac{J_{w x}}{J_{w}}\right) \operatorname{Cov}\left(w^{c}, x\right)\right] \frac{F_{w}}{F} \\
& +\left[\left(-\frac{J_{w w}}{J_{w}}\right) \operatorname{Cov}\left(w^{c}, x\right)+\left(-\frac{J_{w x}}{J_{w}}\right) \operatorname{Var}(x)\right] \frac{F_{x}}{F} \\
& +\int_{E}\left(-\frac{\Delta_{s} J_{w}}{J_{w}}\right) \frac{\Delta_{s} F}{F} h(z, x) \mathbf{I}\{s=i\} \epsilon_{z}(d z)
\end{aligned}
$$

where $F_{w}=\frac{\partial F}{\partial w}, F_{x}=\frac{\partial F}{\partial x}$, and $\frac{\Delta_{s} F}{F}=\delta_{F}(z)$ is the discrete percentage change in the bond price due to a regime shift.

We can clearly see from the above proposition that, in a single regime setting, $\frac{\Delta_{s} F}{F}=0$, $\frac{\Delta_{s} J_{w}}{J_{w}}=0$ and $\frac{\Delta_{s} w}{w}=0$, and (18) is reduced to the standard result in CIR. On the other hand, in the term structure models with regime shifts such as those in Bansal and Zhou (2002) and Evans (2001) among others, $\operatorname{Var}\left(w^{c}\right), \operatorname{Var}(x)$ and $\operatorname{Cov}\left(w^{c}, x\right)$ are assumed to be regime-dependent. Therefore these models have additional econometric flexibility due to the regime-dependent parameters and are shown to have better empirical performance than single-regime models. One implicit assumption maintained by these models, however, is that the regime shift is not a systematic risk to investors, i.e. $\frac{\Delta_{s} J_{w}}{J_{w}}=0$.

To further illustrate the role played by $\frac{\Delta_{s} J_{w}}{J_{w}}$ in determining bond returns, let's assume that $J_{w x}=0$ and $F_{w}=0 .{ }^{7}$ We can obtain from (18) that

$$
\mu_{F}^{*}-r=-\frac{J_{w w}}{J_{w}} w \sigma_{w} \frac{\sigma_{x} F_{x}}{F}-\int_{E} \frac{\Delta_{s} J_{w}}{J_{w}} \frac{\Delta_{s} F}{F} h(z, x) \mathbf{I}\{s=i\} \epsilon_{z}(d z)
$$

The first term in (19) is the instantaneous diffusion risk premium. $\frac{\sigma_{x} F_{x}}{F}$ is the volatility of the bond return due to diffusions in $x(t)$. $\left(-\frac{J_{w w}}{J_{w}}\right) w \sigma_{w}$ measures the excess rate of return per unit of such volatility, commonly referred to as the market price of risk in the literature. The second term is the instantaneous regime-switching risk premium, where $\frac{\Delta_{s} F}{F} h(z, x)$ is the expected discrete percentage change in the bond price due to a shift from regime $i$ to $j$, and $\left(-\frac{\Delta_{s} J_{w}}{J_{w}}\right)$ measures the excess bond return per unit of such expected changes. Hence $\left(-\frac{\Delta_{s} J_{w}}{J_{w}}\right)$ can be analogously defined as the market price of regime-switching risk. And we

\footnotetext{
CIR.

${ }^{7}$ For example, both $J_{w x}=0$ and $F_{w}=0$ hold under log utility function for discount bonds as shown in
} 
can see from (19), unless $J_{w}$ (the marginal utility) is independent of regime $s(t)$, equilibrium bond prices will reflect the risk of regime shifts.

Using Ito's formula, the diffusion term of the bond price is given by $F \sigma_{F}=\left(w \sigma_{w}\right) F_{w}+$ $\sigma_{x} F_{x}$. Hence more insight can be obtained by rewriting (18) as

$$
\mu_{F}^{*}-r=-\operatorname{Cov}\left(\frac{d J_{w}^{c}}{J_{w}}, \frac{d F^{c}}{F}\right)-\int_{E} \frac{\Delta_{s} J_{w}}{J_{w}} \frac{\Delta_{s} F}{F} h(z, x) \mathbf{I}\{s=i\} \epsilon_{z}(d z)
$$

where

$$
\operatorname{Cov}\left(\frac{d J_{w}^{c}}{J_{w}}, \frac{d F^{c}}{F}\right)=\left(\frac{J_{w w} w \sigma_{w}+J_{w x} \sigma_{x}}{J_{w}}\right) \frac{\sigma_{F} F}{F}
$$

(20) implies that the expected excess bond return includes two components under regime shifts. The first term depends on the covariance of the continuous part of the bond return and continuous part of the rate of change in the marginal utility of wealth. The second term depends on the covariation between the discrete percentage change in the marginal utility and the discrete percentage change in the bond price under regime shifts. The higher the covariation, the greater the payoff the bond provides when marginal utility is higher. Hence agents are willing to accept a lower expected rate of return on the asset.

In the previous regime-switching term structure models, greater flexibilities are obtained in fitting the time-varying excess bond returns by forcing $\operatorname{Cov}\left(\frac{d J_{w}^{c}}{J_{w}}, \frac{d F^{c}}{F}\right)$ regime-dependent. Equation (20) shows that allowing investors to price the regime-switching risk (i.e. $\frac{\Delta_{s} J_{w}}{J_{w}} \neq$ 0 ) introduces an additional source of time-variation in the expected excess bond returns. This new component in the bond returns is associated with the potentially large shifts in bond prices across different regimes $\frac{\Delta_{s} F}{F}$. It also depends on the regime-shift intensity $h(z, x)$ as well as the market price of regime-switching risk $-\frac{\Delta_{s} J_{w}}{J_{w}}$. Holding $\frac{\Delta_{s} F}{F}$ and $-\frac{\Delta_{s} J_{w}}{J_{w}}$ constant, the higher the regime-shift intensity $h(z, x)$, the larger the risk premium. ${ }^{8}$ On the other hand, given $\left(-\frac{\Delta_{s} J_{w}}{J_{w}}\right)$ and $h(z, x)$, the regime-switching risk premium depends on the magnitude $\frac{\Delta_{s} F}{F}$. The bigger the difference in the bond price across different regimes, the more important the risk premium due to regime shifts. Empirical results from the previous studies (e.g. Bansal and Zhou, 2002) imply sizeable $\frac{\Delta_{s} F}{F}$, hence suggesting that the regime-switching risk premium is likely to ne an important component of bond returns.

\section{The Term Structure of Interest Rates}

In this section we obtain a closed form solution for the term structure of interest rates. To do so, we assume that $U(c)=\log (c)$. The prices of default-free pure discount bonds are then given by the following proposition (see the Appendix $\mathrm{C}$ for proof).

\footnotetext{
${ }^{8}$ Boudoukh et al (1999) found that economic turning points are usually characterized by highly volatile and strongly time-varying term premium.
} 
Proposition 3 The price at time $t$ of a default-free pure discount bond $F(t, x(t), s(t), T)$ that matures at time $T$ satisfies the following system of partial differential equations

$$
\begin{aligned}
F_{t} & +\left(\mu_{x}-\sigma_{y} \sigma_{x}\right) F_{x}+\frac{1}{2} \sigma_{x}^{2} F_{x x} \\
& +\int_{E}\left(1-\lambda_{s}(z)\right) \Delta_{s} F h(z, x) \mathbf{I}\{s=i\} \epsilon_{z}(d z)=r F
\end{aligned}
$$

with the boundary condition: $f(T, x, s, T)=1$ for all $x$ and $s$.

In the above equation, $F_{t}=\frac{\partial F}{\partial t}, F_{x}=\frac{\partial F}{\partial x}, F_{x x}=\frac{\partial^{2} F}{\partial x^{2}}, \Delta_{s} F=F(t, x(t), s(t-)+\zeta(z), T)-$ $F(t-, x(t-), s(t-), T)$, and $\lambda_{s}(z)=\frac{\delta_{y}(z)}{1+\delta_{y}(z)}$. Note that (21) holds for each regime of $s(t)$, it therefore defines a system of $N$ partial differential equations if there are $N$ distinct regimes. Moreover, under the log utility function, the equilibrium short-term interest rate can be obtained from Proposition 1 as

$$
r=\mu_{y}-\sigma_{y}^{2}+\int_{E} \lambda_{s}(z) h(z, x) \mathbf{I}\{s=i\} \epsilon_{z}(d z)
$$

In general the system (21) does not admit a closed form solution to the bond price. Hence we consider the following affine specification which is known to offer a tractable model of the term structure of interest rate. ${ }^{9}$ In particular, we assume

$$
\begin{aligned}
& \mu_{x}=a_{0}(s)+a_{1}(s) x \\
& \sigma_{x}=\sqrt{\sigma(s) x} \\
& h(z, x)=e^{\eta_{s}(z)} \\
& \sigma_{y}=\theta_{x}(s) \sqrt{\sigma(s) x} \\
& \mu_{y}=x+\theta_{x}^{2}(s) \sigma(s) x-\int_{E} \lambda_{s}(z) \gamma_{m}(d z) \\
& \lambda_{s}(z)=1-e^{\theta_{s}(z)}
\end{aligned}
$$

where $\gamma_{m}(d z)=h(z, x) \mathbf{I}\{s=i\} \epsilon_{z}(d z)$.

Under (23) and (24), the state variable $x(t)$ follows a conventional mean-reverting square-root process with regime-dependent drift and diffusion terms

$$
d x=\left(a_{0}(s)+a_{1}(s) x\right) d t+\sqrt{\sigma(s) x} d B_{t}
$$

Equation (26) and (27) are the assumptions about the drift term and the diffusion term of the physical production process $y(t)$. Under the log utility function, these imply that the market price of the diffusion risk in equilibrium is given by

$$
\lambda_{x}(s)=\theta_{x}(s) \sqrt{\sigma(s) x}
$$

\footnotetext{
${ }^{9}$ Duffie and Kan (1996) and Dai and Singleton (2000) offer detailed discussions of affine term structure models under diffusions. Bansal and Zhou (2002), Evans (2001) and Landen (2000) also use affine specifications in their regime-switching models.
} 
Equation (25) assumes that the Markov chain $s(t)$ has constant transition probabilities given by $e^{\eta_{s}(z)}$ for simplicity. Equation (28) parameterizes the market price of the regimeswitching risk $\lambda_{s}(z)$ as $1-e^{\theta_{s}(z)}$. Moreover (22), (26) and (27) together imply that $r(t)=$ $x(t)$. Therefore we can write

$$
d r=\kappa(s)(\bar{r}(s)-r) d t+\sqrt{\sigma(s) r} d B_{t}
$$

where $\kappa(s)=-a_{1}(s), \bar{r}(s)=\frac{a_{0}(s)}{-a_{1}(s)}$.

The Appendix D shows that, using a log-linear approximation similar to that in Bansal and Zhou (2002), the term structure of interest rates can be solved as follows

Proposition 4 Under assumptions (23) - (28), the price at time $t$ of a default-free pure discount bond with maturity $\tau$ is given by $F(t, \tau)=e^{A(\tau, s(t))+B(\tau, s(t)) r(t)}$ and the $\tau$-period interest rate is given by $R(t, \tau)=-\frac{A(\tau, s(t))}{\tau}-\frac{B(\tau, s(t)) r(t)}{\tau}$, where $A(\tau, s)$ and $B(\tau, s)$ are determined by the following system of differential equations

$$
\begin{aligned}
-\frac{\partial B(\tau, s)}{\partial \tau} & +\left[a_{1}(s)-\theta_{x}(s) \sigma(s)\right] B(\tau, s)+\frac{1}{2} \sigma(s) B^{2}(\tau, s) \\
& +\int_{E}\left(e^{\Delta_{s} A} \Delta_{s} B\right) e^{\eta_{s}(z)+\theta_{s}(z)} \mathbf{1}(s=i) \epsilon_{z}(d z)=1
\end{aligned}
$$

and

$$
-\frac{\partial A(\tau, s)}{\partial \tau}+a_{0}(s) B(\tau, s)+\int_{E}\left(e^{\Delta_{s} A}-1\right) e^{\eta_{s}(z)+\theta_{s}(z)} \mathbf{1}(s=i) \epsilon_{z}(d z)=0
$$

with boundary conditions $A(0, s)=0$ and $B(0, s)=0$, where $\Delta_{s} A=A(\tau, s+\zeta(z))-A(\tau, s)$ and $\Delta_{s} B=B(\tau, s+\zeta(z))-B(\tau, s)$

Proposition 4 nests the models in Bansal and Zhou (2002) and Landen (2000). Without using the log-linear approximation, Landen(2000) only considers models where $\Delta_{s} B=0$ and is silent on the market prices of risk $\theta_{x}(s)$ and $\theta_{s}(z)$. In Bansal and Zhou (2002), the risk of regime shifts is not priced, namely $\theta_{s}(z)$ is assumed to be zero. Admittedly, the model in Proposition 4 still makes some strong assumptions such as constant regime-switching probabilities, even though the theoretical results in the previous section do not depend on these assumptions. In the next section, we provide some empirical results regarding the risk of regime shifts in the term structure of interest rates based on the model above.

\section{Empirical Results}

\subsection{Data and Summary Statistics}

The data used in this study are monthly interest rates from June 1964 to November 2000 obtained from the Center for Research in Security Prices (CRSP). There are eight interest 
rates with maturities ranging from 1 month to 5 years. Table 1 contains their summary statistics. We can see that the yield curve is on average upward-sloping and the large skewness and kurtosis suggest significant departure from Gaussian distribution.

We also report in Table 2 the results from regressions of excess bond returns on forward interest rates and a business cycle dummy variable. We use NBER dates of business cycles to distinguish between expansions and recessions. As in the previous literature we find significant coefficients on forward rates in the regression, suggesting that forward rates contain information about the state variables driving the interest rates. More importantly, the coefficients on the business cycle dummy variable are all negative and significant, suggesting important regime-dependent property of the bond returns. Note that the sign of the coefficient on the business cycle dummy variable is consistent with the counter-cyclical behavior of risk premiums as documented in Fama and French (1989).

\subsection{Econometric Methodology}

We use Efficient Method of Moments ${ }^{10}$ (EMM) estimate the term structure model in Proposition 4 . We assume that there are two distinct regimes $(N=2)$ for $s(t)$. Therefore $(32)$ and (33) define a system of 4 differential equations that must be solved simultaneously. The model has a total of 12 parameters. As in Bansal and Zhou (2002), we fit the model to the data on the 6 -month and the 5 -year rates.

Under EMM procedure, the empirical conditional density of the observed interest rates is first estimated by an auxiliary model that is a close approximation to the true data generating process. Gallant and Tauchen (2001) suggests a semi-nonparametric (SNP) series expansion as a convenient general purpose auxiliary model. As pointed out by Bansal and Zhou (2002), one advantage of using the semi-nonparametric specification for the auxiliary model is that it can asymptotically converge to any smooth distributions (Gallant and Tauchen, 1998), including the density of Markov regime-switching models. The dimension of this auxiliary model can be selected by, for example, the Schwarz's Bayesian Information Criterion (BIC). The score function of the auxiliary model are then used to generate moment conditions for computing a chi-square criterion function, which can be evaluated through simulations given the term structure model under consideration. A nonlinear optimizer is used to find the parameter setting that minimizes the criterion function. Gallant and Tauchen (1996) shows that such estimation procedure yields fully efficient estimators if the score function of the auxiliary model encompasses the score functions of the model under consideration.

\footnotetext{
${ }^{10}$ Bansal et al (1995) and Gallant and Tauchen (1996, 2001) contain detailed discussion of EMM. Bansal and Zhou (2002) is an excellent example of applying EMM to estimate the term structure model under regime shifts. Dai and Singleton (2000) also provides extensive discussions of estimating affine term structure models using EMM procedure.
} 


\subsection{Results}

Table 3 and Table 4 contain the results from SNP estimation. In searching for the preferred specification, we follow closely Bansal and Zhou (2002) mainly because we use the same interest rate data (with longer sample period). Different choices of SNP density and their corresponding BIC values are reported in Table 3. Consistent with Bansal and Zhou (2002), we find that the SNP specification with 1 lag $\left(L_{\mu}=1\right)$ in the VAR-based conditional mean, 5 lags in ARCH term $\left(L_{r}=5\right)$ and a polynoinal of order $4\left(K_{z}=4\right)$ in the standardized residual $z$ has the overall best fit based on BIC. Estimates of the coefficients in the preferred SNP density are reported in Table 4.

Given the SNP density, the parameters of the term structure model can be estimated through simulations. We estimate three versions of CIR model. Model One is the standard one-factor CIR model. Model 2 is a one-factor CIR model with regime shifts, but the risk of regime shifts is not priced. We allow the parameters in the diffusion process of the instantaneous short-term interest rate $r(t)$ to be regime-dependent. We also assume different market prices of the diffusion risk across regimes. Model 3 is the one-factor CIR developed in Proposition 4 where the risk of regime shifts is priced. The results are reported in Table $5 .^{11}$

In the standard 1-factor CIR model, the parameter estimates imply a highly persistent short-term interest rate process with a long-run average level of $6.4 \%\left[\bar{r}=a_{0} /\left(-a_{1}\right)\right]$ and a speed of adjustment of only $0.0907\left(\kappa=-a_{1}\right)$. This roughly corresponds an AR(1) process with the coefficient on the lagged interest rate being around 0.91 , which is consistent with the results from the previous empirical studies of the interest rate. The estimates also imply stochastic volatility is an important property of the interest rate process with a conditional standard deviation of $1.27 \%$ on average $(\sqrt{\sigma \bar{r}})$.

Model 2 introduces Markov regime shifts into the standard CIR model without pricing such risk. Consistent with the previous studies, we find that the interest rate process is characterized by two distinct regimes. In one regime (Regime 2 ) the short rate $r(t)$ is highly persistent $\left(\kappa=-a_{1}=0.0735\right)$ with a long-run mean of $11.7 \%\left[\bar{r}=a_{0} /\left(-a_{1}\right)\right]$. The short rate in the other regime (Regime 1$)$, however, is less persistent $\left(\kappa=-a_{1}=0.1501\right)$ with a much lower long-run mean of $1.8 \%$. Given the average short-term interest rate of $5 \%-6 \%$ in the sample (see Table 1), this implies that the interest rate is usually rising in Regime 2 and declining in Regime 1. This empirical regularity is consistent with features of the business cycle where expansions are usually characterized by rising interest rates and recessions tend to witness declining interest rates. Moreover, the estimated coefficients on the conditional volatility and the market price of (diffusion) risk all are different across regimes, further

\footnotetext{
${ }^{11}$ We didn't estimate a multi-factor model mainly because the main purpose of this section is not to obtain a good empirical fitting, but to highlight the potential impact of the systematic risk of regime shifts on the term structure of interest rates. Bansal and Zhou (2002) and Dai et al (2003) provide excellent estimation results of multi-factor term structure models under regime shifts.
} 
suggesting that the yield curve exhibits strong regime-dependence properties. ${ }^{12}$ The regimeshift intensity is parameterized as $e^{\eta_{s}(z)}$ in the model. Table 5 reports that $\eta_{s}(1,2)=$ -1.2040 and $\eta_{s}(2,1)=-1.4706$. This implies that Regime 2 is more persistent than Regime 1 (namely smaller transition probability from Regime 2 to Regime 1). For the monthly data used in the paper, the estimates of $\eta_{s}(z)$ suggest that the probability of switching from Regime 1 to Regime 2 is approximately 0.0250 while the probability of switching from Regime 2 to Regime 1 is approximately 0.0191. These findings are consistent with the results based on discreet time models (e.g. Bansal and Zhou, 2002).

In Model 3, the risk of regime shifts is priced. The estimates of the model parameters $\left(a_{0}\right.$, $a_{1}, \sigma, \theta_{x}$ and $\left.\eta_{s}\right)$ are similar to those obtained in Model 2, confirming that the periodic shifts across distinct regimes is an important empirical property of the interest rate dynamics. In Model 3, the implied long-run mean of $r(t)$ becomes $11.1 \%$ in Regime 2 and $2.4 \%$ in Regime 1 respectively. The estimates of $a_{1}$ and $\sigma$ indicate that $r(t)$ is more persistent with a larger conditional variance in Regime $2\left(\kappa=-a_{1}=0.0916, \sigma=0.0034\right)$ than in Regime $1\left(\kappa=-a_{1}=0.1491, \sigma=0.0025\right)$. As in Model 2, we also find that the market price of the diffusion risk varies across regimes. The estimate of $\theta_{x}$ is -15.54 in Regime 1 and -17.00 in Regime 2 respectively. Finally, the estimates of $\eta_{s}(z)$ imply that, at monthly frequency, the Markov chain $s(t)$ switches from Regime 1 to Regime 2 with probability 0.0260 and switches from Regime 2 to Regime 1 with probability 0.0169 .

In Figure 1, we plot the yield curve at the estimated parameters in Regime 1 together with that in Regime 2 by fixing the short-term interest rate at the sample average of $5.6 \%$ using Model 3. The differences in the yield curves are obvious. In Regime 2 the yield curve is higher and steeper compared to that in Regime 1 due to the fact that interest rates tend to rise in Regime 2. The average yield curve in Regime 1 not only has a lower level, but also has a different shape. It initially slopes downward and then slopes upward. This is because that the short-term interest rate declines in Regime 1 on average. Since the term premiums are small for bonds of short maturities, the interest rates on these bonds are mainly determined by the expectation of the short-term interest rate in the near future, therefore resulting in a negative slope in the yield curve. However, as maturities of the bonds increase, the term premiums start to play a more important role in determining the interest rates. Moreover, since Regime 1 is not as persistent as Regime 2, the short-term interest rate is also expected to increase over a long horizon as $s(t)$ switches from Regime 1 to Regime 2 in the future. Hence the slope of the yield curve becomes positive as maturities increase.

Figure 1 clearly shows that the yield curve alternates between two distinct regimes as $s(t)$ evolves over time. Whether or not these regime shifts pose a significant risk to investors depends on the estimate of $\theta_{s}(z)$, the parameter that determines the market price of the

\footnotetext{
${ }^{12}$ The conditional volatility of the short term interest rate $r(t)$ is given by $\sqrt{\sigma r(t)}$, and the market price of diffusion risk is given by $\theta_{x} \sqrt{\sigma r(t)}$ in the model.
} 
regime-switching risk. ${ }^{13}$ Table 5 shows that the estimates of $\theta_{s}(z)$ are highly significant in both regimes. In particular, $\theta_{s}(z)$ is estimated to be 0.1438 with a standard error of 0.0337 in Regime 1 and -0.0789 with a standard error of 0.0109 in Regime 2 respectively. Moreover there is a substantial improvement in the goodness-of-fit of the regime-switching model. The $\chi^{2}$ statistics decreases from 109.70 with 18 degree of freedom in Model 2 to 60.38 with 16 degree of freedom after the regime-switching risk is priced in Model 3.

Notice that the $\chi^{2}$ statistics still indicates an overall poor fitting of the model. This is not surprising given that the model has only one factor (plus a Markov regime-switching variable). Bansal and Zhou (2002) and Dai et al. (2003) showed that the overall fit can be further improved in multi-factor models. Moreover, the results in Dai et al. (2003) indicate that the market price of regime-switching risk remains significant even when additional factors are included. These results suggest that the significant regime-switching risk parameter found in our model is not due to omitted factors. The regime shifts in the term structure documented in many empirical studies most likely represent a systematic risk.

In fact, following the approach in Bansal and Zhou (2002), ${ }^{14}$ we can plot the implied regimes together with the business cycle expansions and recessions identified by NBER in Figure 2. The figure clearly shows that the distinct regimes underlying the dynamics of the term structure of interest rates are intimately related to the fluctuations of the aggregate economy. Therefore it is important that these periodic shifts in regimes are treated as a systematic risk to investors in the dynamic term structure models. It is also interesting to note from Figure 7 that the regime shifts tend to precede business cycle turning points. ${ }^{15}$ This is consistent with the empirical finding that the yield curve has significant predictive power for the business cycle, see Estrella and Mishkin (1995) and Chauvet and Potter (2003) among others.

\subsubsection{The Term Premiums due to Regime-switching Risk}

To assess quantitatively the potential impact of the regime-switching risk, we can decompose the term premium on a long-term bond into two parts. One is a term premium due to the diffusion risk and the other component is associated with the risk of regime shifts. In

\footnotetext{
${ }^{13}$ The market price of the regime switching risk $\lambda_{s}(z)$ is parameterized as $1-e^{\theta_{s}(z)}$ in the model, see equation (28).

${ }^{14}$ Specifically, the estimated term structure model allows us to compute interest rates of different maturities conditional on the regime $\hat{R}\left(t, \tau \mid s_{t}\right)$. An estimate of $s_{t}$ can be obtained by choosing the regime that minimizes the average difference between the actually observed interest rate $R(t, \tau)$ and $\hat{R}\left(t, \tau \mid s_{t}\right)$, i.e. $\hat{s}_{t}=\arg \min \sum_{\tau}\left|R(t, \tau)-\hat{R}\left(t, \tau \mid s_{t}\right)\right|$

${ }^{15}$ Note that the regime shifts near the end of the sample period precede the most recent recession starting from March 2001 as classified by NBER.
} 
particular, ignoring the Jensen's inequality terms, one can obtain that,

$$
\begin{aligned}
& R\left(t_{0}, \tau\right)-\frac{1}{\tau} E_{t_{0}}\left[\int_{t_{0}}^{t_{0}+\tau} r_{t} d t\right] \approx \\
& \frac{1}{\tau} E_{t_{0}}\left[\int_{t_{0}}^{t_{0}+\tau} \theta_{x}\left(s_{t}\right) \sigma\left(s_{t}\right) x_{t} B\left(t_{0}+\tau-t, s_{t}\right) d t\right]+ \\
& \frac{1}{\tau} E_{t_{0}}\left[\int_{t_{0}}^{t_{0}+\tau} \int_{E} \lambda_{s}(z)\left(e^{\Delta_{S} A\left(t_{0}+\tau-t, s_{t}\right)+\Delta_{s} B\left(t_{0}+\tau-t, s_{t}\right) x_{t}}-1\right) \gamma_{m}(d t, d z)\right]
\end{aligned}
$$

where $R\left(t_{0}, \tau\right)$ is the interest rate on a default-free bond of maturity $\tau$ at time $t_{0}, r_{t}$ is the instantaneous short-term interest rate. So the left-hand side of (34) is the difference between the interest rate on the long-term bond of maturity $\tau$ and the average of the expected future short-term interest rate. The Expectation Hypothesis of the term structure maintains that this difference is zero because long-term interest rates are solely determined by the expected future short rate. However, as it is made clear in (34), long-term interest rates can deviate substantially from the levels implied by the Expectation Hypothesis. The first term on the right-hand of (34) is the excess return demanded by investors due to the diffusion risk and the second term is the excess return due to the regime-switching risk, where the market prices of risk are given by $\theta_{x}\left(s_{t}\right) \sqrt{\sigma\left(s_{t}\right) x_{t}}$ and $\lambda_{s}(z)$ respectively. Note that $A(\tau, s)$ and $B(\tau, s)$ are determined in Proposition 4 and $\gamma_{m}(d t, d z)$ is given in (2). Using the estimated parameters, the average values of these two components of the term premium can be obtained through Monte Carlo simulations. ${ }^{16}$ In Figure 3, we report the average total term premiums and the two components for bonds of various maturities ranging from 3 months to 30 years. In each graph the lower part measures the diffusion risk premiums and the upper part measures the regime-switching risk premiums. For bonds with short maturities (less than 1-year), we can see that the term premiums are small and are due to the diffusion risk. As bond maturity increases, both components of the term premiums increase, and the regime-switching risk premiums become a significant part of the total term premiums. The lower panel of Figure 2 indicates that the regime-switching risk can account for more than $10 \%$ of the term premiums for bonds with maturities longer than 6 years, and up to $15 \%$ for a 30 -year bond.

\subsubsection{How Does the Regime-shift Risk Affect the Yield Curve?}

The term structure model developed in Proposition 4 allows us to compute the prices of bonds of different maturities in the presence of the regime-switching risk. In this section we examine the impact of such risk on the yield curve. Specifically, we compare the estimated bond prices in Model 3 with the bond prices obtained using the same model and the same

\footnotetext{
${ }^{16}$ More specifically, we simulate the 5000 independent sample paths of $r(t)$ and $s(t)$ given $r\left(t_{0}\right)$ and $s\left(t_{0}\right)$. To get ride of the impact of the initial values of $r\left(t_{0}\right)$ and $s\left(t_{0}\right)$, for each sample path the first one thousand points of $r(t)$ and $s(t)$ are ignored. We then take the average over the 5000 sample paths of $r(t)$ and $s(t)$ to obtain unconditional expectations.
} 
parameter values except holding the market price of the regime-switching risk $\theta_{s}(z)$ at zero. ${ }^{17}$ When computing the bond prices, we set the short-term interest rate at the sample average of the 1-month rate of $5.6 \%$. We report the price differential between the two regimes in Figure 4 and the corresponding yield curves in Figure 5. Consistent with the result from the last section, we find that when fixing $\theta_{s}(z)$ at zero, the prices of long-term bonds become significantly higher because of the reduction of the (total) term premiums. Figure 4 shows that, for example, the price of a 30 -year bond would be about $15 \%$ higher if $\theta_{s}(z)$ is set to zero instead of its estimated value of 0.1438 in Regime 1 or -0.0789 in Regime 2. On the other hand, the regime-switching risk has almost no effect on short-term bonds. These result implies that the regime-shift risk mostly affects the long end of the yield curve as expected. Ignoring the regime-shift risk would lead to underestimation of long-term interest rates, and therefore flatter yield curves (see Figure 5).

\section{Concluding Remarks}

Previous studies have provided strong empirical evidence that the joint movement of interest rates of different maturities can be well described by dynamic term structure models that incorporate regime shifts. Moreover these studies also show that there is a close link between the regime shifts and the business cycle fluctuations. Therefore it is very likely that such large periodic shifts of interest rates across distinct regimes present a systematic risk to investors. This paper elucidates such regime-switching risk in a general equilibrium model of the term structure of interest rates. We show that the regime-switching risk premium depends on the covariations between the discreet changes in marginal utility and bond prices. This new component therefore introduces an additional source of time-variation in bond risk premiums and can offer additional econometric flexibility to account for the joint movements of interest rates with different maturities. Our empirical results suggest that the regime-switching risk is a significant factor determining bond prices and has a major impact particular on the long-end of the yield curve.

One caveat of the current paper is that we have to rely on log-linear approximations to obtain a closed-form solution to the term structure of interest rates. How important the approximation errors are is a question we are currently investigating. Nevertheless, we notice that relaxing some of the assumptions (such as regime-dependent factor loadings) can lead to exact solutions of the yield curve. Another caveat to note is that the estimated 1 -factor term structure model has a poor empirical fitting. We do not pretend that the term structure is fully characterized by this single factor. We use that model mainly to illustrate the potential impact of a systematic regime-switching risk on the yield curve.

This systematic risk of regime shifts is also likely to have important implications for pricing bond derivatives (e.g. Singleton and Umantsev, 2002) as well as for investors'

\footnotetext{
${ }^{17}$ In Model 3, the estimates of $\theta_{s}(z)$ are 0.1438 with a standard error of 0.0337 in Regime 1 and -0.0789 with a standard error of 0.0109 in Regime 2 respectively.
} 
optimal portfolio choice problem (e.g. Campbell and Viceira, 2001). These are left for future research. Another extension is to estimate the term structure model jointly with macroeconomic variables under regime shifts. This would provide more direct evidence regarding the nature of the regime-switching risk. 


\section{A Proof of Proposition 1}

Let $J(w(t), s(t), x(t))=\sup E_{t}\left[\int_{t}^{\infty} e^{-\rho(\tau-t)} U(c(\tau)) d \tau\right]$. We assume that a solution to the agent's problem exists and indirect utility function $J(w(t), s(t), x(t))$ as well as the optimal consumption and portfolio choice satisfy the Bellman equation (see the result in Section 3.1.6 of Kushner and Dupuis, 2001)

$$
\sup _{\left(\phi_{1}, \phi_{2}, c\right)} \mathcal{D} J(w, s, x)-\rho J(w, s, x)+U(c)=0
$$

where

$$
\mathcal{D} J(w, s, x)=\left(w \mu_{w}\right) J_{w}+\mu_{x} J_{x}+\frac{1}{2}\left(w \sigma_{w}\right)^{2} J_{w w}+\left(w \sigma_{w}\right) \sigma_{x} J_{w x}+\frac{1}{2} \sigma_{x}^{2} J_{x x}+\int_{E} \Delta_{s} J \gamma_{m}(d z)
$$

and

$$
\Delta_{s} J=J\left(w\left(1+\delta_{w}(z)\right), s+\zeta(z), x\right)-J(w, s, x)
$$

In the above equations, $J_{w}=\frac{\partial J}{\partial w}, J_{x}=\frac{\partial J}{\partial x}, J_{w w}=\frac{\partial^{2} J}{\partial w^{2}}, J_{w x}=\frac{\partial^{2} J}{\partial w \partial x} \cdot \mu_{w}, \sigma_{w}$ and $\delta_{w}$ are given in (12), (13) and (14) respectively. $\gamma_{m}(d z)$ is defined in (17). $\mu_{x}$ and $\sigma_{x}$ are the drift and diffusion terms of the state variable $x(t)$ respectively as defined in (1).

The first order conditions (35) are

$$
\begin{gathered}
U^{\prime}(c)-J_{w}=0 \\
w\left(\mu_{y}-r\right) J_{w}+\left(w \sigma_{w}\right)\left(w \sigma_{y}\right) J_{w w}+\left(w \sigma_{y}\right) \sigma_{w} J_{w x} \\
+\int_{E} w \delta_{y}(z) J_{w}\left(w\left(1+\delta_{w}(z)\right), s+\zeta(z), x\right) \gamma_{m}(d z)=0 \\
w\left(\mu_{F}-r\right) J_{w}+\left(w \sigma_{w}\right)\left(w \sigma_{F}\right) J_{w w}+\left(w \sigma_{F}\right) \sigma_{x} J_{w x} \\
+\int_{E} w \delta_{F}(z) J_{w}\left(w\left(1+\delta_{w}(z)\right), s+\zeta(z), x\right) \gamma_{m}(d z)=0
\end{gathered}
$$

Note that at equilibrium $\phi_{1}=1$ and $\phi_{2}=0$, hence Proposition 1 follows from (37).

\section{B Proof of Proposition 2}

From (38) above, we have

$$
\begin{aligned}
\mu_{F}+\int_{E} \delta_{F}(z) \gamma_{m}(d z)-r & =\left(-\frac{J_{w w}}{J_{w}}\right) \frac{\left(w \sigma_{w}\right)\left(w \sigma_{F}\right)}{w}+\left(-\frac{J_{w x}}{J_{w}}\right) \frac{\left(w \sigma_{F}\right) \sigma_{x}}{w} \\
& +\int_{E}\left(-\frac{\Delta_{s} J_{w}}{J_{w}}\right) \frac{\Delta_{s} F}{F} \gamma_{m}(d z)
\end{aligned}
$$


Apply Ito's formula to $F(t, w, s, x)$, we have ${ }^{18}$

$$
\begin{aligned}
d F & =\left[F_{t}+\left(w \mu_{w}\right) F_{w}+\mu_{x} F_{x}+\frac{1}{2}\left(w \sigma_{w}\right)^{2} F_{w w}+\left(w \sigma_{w}\right) \sigma_{x} F_{w x}+\frac{1}{2} \sigma_{x}^{2} F_{x x}\right] d t \\
& +\left[\left(w \sigma_{w}\right) F_{w}+\sigma_{x} F_{x}\right] d B(t)+\int_{E} \Delta_{s} F m(d t, d z)
\end{aligned}
$$

Compare (40) with (7), we have

$$
\begin{gathered}
F \mu_{F}=F_{t}+\left(w \mu_{w}\right) F_{w}+\mu_{x} F_{x}+\frac{1}{2}\left(w \sigma_{w}\right)^{2} F_{w w}+\left(w \sigma_{w}\right) \sigma_{x} F_{w x}+\frac{1}{2} \sigma_{x}^{2} F_{x x} \\
F \sigma_{F}=\left(w \sigma_{w}\right) F_{w}+\sigma_{x} F_{x}
\end{gathered}
$$

and

$$
\delta_{F}(z)=\frac{\Delta_{s} F}{F}
$$

Proposition 2 follows after substituting (42) and (43) into (39) and defining $\mu_{F}^{*}=\mu_{F}+$ $\int_{E} \delta_{F}(z) \gamma_{m}(d z)$.

\section{Proof of Proposition 3}

Proposition 2 implies that

$$
\begin{aligned}
& \mu_{F}+\int_{E} \delta_{F}(z) \gamma_{m}(d z)-r \\
& =\left[\left(-\frac{J_{w w}}{J_{w}}\right) \operatorname{Var}(w)+\left(-\frac{J_{w x}}{J_{w}}\right) \operatorname{Cov}(w, x)\right] \frac{F_{w}}{F} \\
& +\left[\left(-\frac{J_{w w}}{J_{w}}\right) \operatorname{Cov}(w, x)+\left(-\frac{J_{w x}}{J_{w}}\right) \operatorname{Var}(x)\right] \frac{F_{x}}{F} \\
& +\int_{E}\left(-\frac{\Delta_{s} J_{w}}{J_{w}}\right) \frac{\Delta_{s} F}{F} \gamma_{m}(d z)
\end{aligned}
$$

Using (41), we have

$$
\begin{aligned}
F_{t} & +\frac{1}{2}\left(w \sigma_{w}\right)^{2} F_{w w}+\left(w \sigma_{w}\right) \sigma_{x} F_{w x}+\frac{1}{2} \sigma_{x}^{2} F_{x x} \\
& +\left[\mu_{x}-\left(-\frac{J_{w w}}{J_{w}}\right)\left(w \sigma_{w}\right) \sigma_{x}-\left(-\frac{J_{w x}}{J_{w}}\right) \sigma_{x}^{2}\right] F_{x} \\
& +\left[\left(w \mu_{w}\right)-\left(-\frac{J_{w w}}{J_{w}}\right)\left(w \sigma_{w}\right)^{2}-\left(-\frac{J_{w x}}{J_{w}}\right)\left(w \sigma_{w}\right) \sigma_{x}\right] F_{w} \\
& +\int_{E}\left(1+\frac{\Delta_{s} J_{w}}{J_{w}}\right) \Delta_{s} F \gamma_{m}(d z)=r F
\end{aligned}
$$

\footnotetext{
${ }^{18}$ See Protter (1990) for the generalized Ito's formula for semi-martingales.
} 
Note again that at equilibrium, $\phi_{1}=1$ and $\phi_{2}=0$. Hence (12), (13) and (14) imply that

$$
\begin{array}{r}
w \mu_{w}=w \mu_{y}-c \\
w \sigma_{w}=w \sigma_{y} \\
w \delta_{w}(z)=w \delta_{y}(z)
\end{array}
$$

Moreover, Proposition 1 implies

$$
\begin{aligned}
w r & =w \mu_{y}-\left(-\frac{J_{w w}}{J_{w}}\right)\left(w \sigma_{y}\right)^{2}-\left(-\frac{J_{w x}}{J_{x}}\right)\left(w \sigma_{y}\right) \sigma_{x} \\
& +\int_{E}\left(1+\frac{\Delta_{s} J_{w}}{J_{w}}\right) w \delta_{y}(z) \gamma_{m}(d z)
\end{aligned}
$$

Combining (45) - (49), We have the following fundamental partial differential equations of asset pricing as in CIR

$$
\begin{aligned}
F_{t} & +\frac{1}{2}\left(w \sigma_{y}\right)^{2} F_{w w}+\left(w \sigma_{y}\right) \sigma_{x} F_{w x}+\frac{1}{2} \sigma_{x}^{2} F_{x x} \\
& +\left[\mu_{x}-\left(-\frac{J_{w w}}{J_{w}}\right)\left(w \sigma_{y}\right) \sigma_{x}-\left(-\frac{J_{w x}}{J_{w}}\right) \sigma_{x}^{2}\right] F_{x} \\
& +\left[w r-c-\int_{E}\left(1+\frac{\Delta_{s} J_{w}}{J_{w}}\right) w \delta_{y}(z) \gamma_{m}(d z)\right] F_{w} \\
& +\int_{E}\left(1+\frac{\Delta_{s} J_{w}}{J_{w}}\right) \Delta_{s} F \gamma_{m}(d z)=r F
\end{aligned}
$$

Under logarithm utility function $U(c(t))=\log c(t)$, it is well known that the indirect utility function is separable in $w(t)$ and $x(t)$ and $s(t)$, i.e. $J(w, s, x)$ can be written as $\frac{1}{\rho} \log w+f(s, x)$ where $f(s, x)$ solve the system of differential equation after substituting $J(w, s, x)$ and the optimal choice of consumption $\left(c^{*}\right)$ and portfolio $\left(\phi_{1}^{*}, \phi_{2}^{*}\right)$ into the Bellman equation (35). This separability implies that $J_{w x}=0$.

Moreover, for default-free discount bonds, $F_{w}=0, F_{w w}=0$ and $F_{w x}=0$. Therefore equation (50) can simplified as

$$
F_{t}+\frac{1}{2} \sigma_{x}^{2} F_{x x}+\left(\mu_{x}-\sigma_{y} \sigma_{x}\right) F_{x}+\int_{E}\left(1+\frac{\Delta J_{w}}{J_{w}}\right) \Delta_{s} F \gamma_{m}(d z)=r F
$$

Using the fact that $J_{w}=\frac{1}{\rho w}$ and (48), it can be easily shown

$$
1+\frac{\Delta_{s} J_{w}}{J_{w}}=1-\lambda_{s}(z)
$$


where $\lambda_{s}(z)=\frac{\delta_{y}(z)}{1+\delta_{y}(z)}$.

Proposition 3 can be obtained by substituting the above equation into (51). Note that (51) defines a system of partial differential equations.

\section{Proof of Proposition 4}

Without loss of generality, let the price at time $t$ of a pure-discount bond that will mature at $T$ be given as

$$
F(t, s(t), r(t), T)=e^{A(\tau, s(t))+B(\tau, s(t)) r(t)}
$$

where $\tau=T-t$ and $A(0, s)=0, B(0, s)=0$.

Proposition 3 then implies

$$
\begin{aligned}
r & =-\frac{\partial A(\tau, s)}{\partial \tau}-\frac{\partial B(\tau, s)}{\partial \tau} r \\
& +\left[a_{0}(s)+\left(a_{1}(s)-\theta_{x}(s) \sigma(s)\right) r\right] B(\tau, s)+\frac{1}{2}[\sigma(s) r] B^{2}(\tau, s) \\
& +\int_{E}\left(e^{\Delta_{s} A+\Delta_{s} B r}-1\right) e^{\eta_{s}(z)+\theta_{s}(z)} \mathbf{1}(s=i) \epsilon_{z}(d z)
\end{aligned}
$$

where $\Delta_{s} A=A(\tau, s+\zeta(z))-A(\tau, s)$ and $\Delta_{s} B=B(\tau, s+\zeta(z))-B(\tau, s)$

Since $r$ is small, applying log-linear approximation to $e^{\Delta_{s} B r}$, we have

$$
e^{\Delta_{s} B r} \approx 1+\Delta_{s} B r
$$

Proposition 4 follows by substituting the above equation into (53) and match the coefficients on $r$ on both side of the equation. 


\section{References}

[1] Ahn, C. M. and H. E. Thompson (1988), "Jump-Diffusion Processes and the Term Structure of Interest Rates", The Journal of Finance 43, 155-174.

[2] Ang, A. and G. Bekaert (1998), "Regime Switches in Interest Rates", NBER working paper 6508 .

[3] Bansal, R. and H. Zhou (2002), "Term Structure of Interest Rates with Regime Shifts", The Journal of Finance 57, 1997-2043.

[4] Bansal, R., R. Gallant and G. Tauchen (1995), "Nonparametric Estimation of Structural Models for High-frequency Currency Market Data", Journal of Econometrics 66, 251-287.

[5] Bielecki, T. and M. Rutkowski (2001), "Modeling of the Defaultable Term Structure: Conditional Markov Approach", Working paper, The Northeastern Illinois University.

[6] Boudoukh, Jacob et al. (1999), "Regime Shifts and Bond Returns", Working paper, New York University.

[7] Campbell, J. Y. and L. M. Viceira (2001), "Who Should Buy Long-Term Bonds", The American Economic Review 91, 99-127.

[8] Chauvet, M. and S. Potter (2003), "Forecasting Recessions Using the Yield Curve", Journal of Forecasting, forthcoming.

[9] Cox, J., J. Ingersoll and S. Ross (1985a), "An Interemporal General Equilibrium Model of Asset Prices", Econometrica 53, 363-384.

[10] Cox, J., J. Ingersoll and S. Ross (1985b), "A Theory of the Term Structure of Interest Rates", Econometrica 53, 385-407.

[11] Dai Q. and K. Singleton (2000), "Specification Analysis of Affine Term Structure Models", Journal of Finance LV, 1943-1978.

[12] Dai, Q. and K. Singleton (2003), "Term Structure Dynamics in Theory and Reality", The Review of Financial Studies 16, 631-678

[13] Dai, Q., K. J. Singleton and W. Yang (2003), "Regime Shifts in a Dynamic Term Structure Model of U.S. Treasury Bond Yields", working paper, New York University.

[14] Diebold, F. and G. Rudebusch (1996), "Measuring Business Cycles: A Modern Perspective", Review of Economics and Statistics 78, 67-77.

[15] Duffie, D. and R. Kan (1996), "A Yield-Factor Model of Interest Rates", Mathematical Finance 6, 379-406. 
[16] Duffie, D., J, Pan and K. Singleton (2000), "Transform Analysis and Asset Pricing for Affine Jump-Diffusions", Econometrica 68, 1343-1376.

[17] Elliott, R. J. and R. S. Mamon, "Term structure of a Vasicek model with a Markovian mean reverting level", Working paper, University of Calgary.

[18] Estrella, Arturo and Federic S. Mishkin (1995), "The Term Structure of Interest Rates and Its Role in Monetary Policy for the European Central Bank", NBER working paper, No.5279.

[19] Evans, M. (2001), "Real Risk, Inflation Risk, and the Term Structure", Working paper, Georgetown University.

[20] Filardo, A.J. (1994), "Business Cycle Phases and Their Transitional Dynamics", Journal of Business and Economic Statistics 12, 299-308.

[21] Garcia, R. and P. Perron (1996), "An Analysis of the Real Interest Rate Under regime Shifts", Review of Economics and Statistics 78, 111-125.

[22] Gallant, R. and G. Tauchen (1996), "Which moment to match?", Econometric Theory $12,657-681$.

[23] Gallant, R. and G. Tauchen (1998), "Reprojecting Partially Observed System with Application to Interest Rate Diffusions", Journal of the American Statistical Association 93, 10-24.

[24] Gallant, R. and G. Tauchen (2001), "Efficient Method of Moments", manscript.

[25] Gray, S. (1996), "Modeling the Conditional Distribution of Interest Rates as a RegimeSwitching Process", Journal of Financial Economics 42, 27-62.

[26] Hamilton, J. (1988), "Rational Expectations Econometric Analysis of Changes in Regimes: An Investigation of the Term Structure of Interest Rates", Journal of Economic Dynamics and Control 12, 385-423.

[27] Hamilton, J. (1989), "A New Approach to the Economic Analysis of Nonstationary Time Series and the Busines Cycle", Econometrica 57, 357-384.

[28] Kushner, H. J. and P. Dupuis (2001), "Numerical Methods for Stochastic Control Problems in Continuous Time", 2nd edition, Springer, New York.

[29] Landen, C. (2000), "Bond Pricing in a Hidden Markov Modle of the Short Rate", Finance and Stochastics 4, 371-389.

[30] Last, G. and A. Brandt (1995), "Marked Point Processes on the Real Line", New York: Springer. 
[31] Naik, V. and M. H. Lee (1997), "Yield Curve Dynamics with Discrete Shifts in Economic Regimes: Theory and Estimation", Working paper, University of British Columbia.

[32] Protter, P. (1990), "Stochastic Intergration and Differential Equations", Berling: Springer Verlag.

[33] Singleton, K. J. and L. Umantsev (2002), "Pricing Coupon-Bond Options and Swaptions in Affine Term Structure Models", Mathematical Finance 12, 427-446. 
Table 1 Summary Statistics of the term structure of interest rates 1964 - 2000 (the first column indicates the maturities of the interest rates)

\begin{tabular}{|l|r|r|r|r|r|r|r|}
\hline & \multicolumn{1}{l}{ mean } & \multicolumn{1}{l}{ std } & \multicolumn{1}{l|}{ corr } & \multicolumn{1}{l|}{ skew } & \multicolumn{1}{l|}{ kurt } & \multicolumn{1}{l|}{$\max$} & \multicolumn{1}{l|}{ in } \\
\hline 1-month & 0.05608 & 0.025452 & 1 & 1.28697 & 5.17923 & 0.161376 & 0.013176 \\
\hline 3-month & 0.060275 & 0.026706 & 0.985712 & 1.26099 & 4.91011 & 0.160332 & 0.017112 \\
\hline 6-month & 0.062443 & 0.026954 & 0.978383 & 1.21604 & 4.69365 & 0.165168 & 0.017808 \\
\hline 1-year & 0.06455 & 0.026314 & 0.964593 & 1.07663 & 4.21845 & 0.15812 & 0.01956 \\
\hline 2-year & 0.066561 & 0.025774 & 0.938704 & 1.01143 & 3.93613 & 0.15639 & 0.02368 \\
\hline 3-year & 0.068159 & 0.025069 & 0.916046 & 0.998881 & 3.84814 & 0.15557 & 0.0296 \\
\hline 4-year & 0.069354 & 0.024795 & 0.897531 & 0.965216 & 3.74017 & 0.15824 & 0.03118 \\
\hline 5-year & 0.069981 & 0.024589 & 0.883406 & 0.921516 & 3.57469 & 0.15001 & 0.03346 \\
\hline
\end{tabular}

Note: mean = average interest rate; std $=$ standard deviation; corr = correlation coefficient between long rates and the 1-month interest rate; skew = skewness; kurt = kurtosis; $\max =$ maximum interest rate; min=minimum interest rate 


\section{Table 2: Using forward rates to predict excess bond returns.}

\begin{tabular}{|c|c|c|c|c|c|c|}
\hline & 3-month 6-month & 1-year & 2-year & 3-year & 4-year & 5-year \\
\hline \multirow[t]{2}{*}{ constant } & \begin{tabular}{|l|l|}
0.0030 & 0.0116 \\
\end{tabular} & 0.0188 & 0.0224 & 0.0236 & 0.0187 & 0.016 \\
\hline & \begin{tabular}{|l|l}
$(0.0022)$ & $(0.0054)$
\end{tabular} & $(0.0124)$ & $(0.0227)$ & $(0.0321)$ & $(0.0321)$ & $(0.0455)$ \\
\hline \multirow[t]{2}{*}{ Bus-cycle } & \begin{tabular}{l|l}
-0.0074 & -0.0167 \\
\end{tabular} & -0.0313 & -0.0495 & -0.0619 & -0.0694 & -0.0773 \\
\hline & \begin{tabular}{|l|l}
$(0.0021)$ & $(0.0050)$
\end{tabular} & $(0.0111)$ & $(0.0199)$ & $(0.0272)$ & $(0.0272)$ & $(0.0371$ \\
\hline \multirow[t]{2}{*}{ | } & \begin{tabular}{l|l}
-0.2145 & -0.5015 \\
\end{tabular} & -0.1386 & -0.8860 & -1.4536 & -1.9372 & -2.2324 \\
\hline & $(0.0979) \quad(0.2362)$ & (0.5121) & $(0.8805)$ & $(1.1746)$ & $(1.1746)$ & (1.7405) \\
\hline \multirow[t]{2}{*}{6} & $0.1363 \quad 0.3895$ & -0.2358 & -0.0988 & -0.0620 & -0.3291 & -0.6753 \\
\hline & 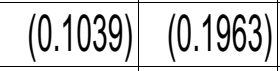 & $(0.4121)$ & $(0.7022)$ & $(0.9602)$ & $(0.9602)$ & $(1.4695)$ \\
\hline \multirow[t]{2}{*}{160} & \begin{tabular}{l|l}
0.1935 & 0.2128
\end{tabular} & 0.5717 & 1.2403 & 1.8126 & 2.6277 & 3.3306 \\
\hline & $(0.0694)(0.1458)$ & $(0.3320)$ & $(0.6012)$ & $(0.8451)$ & $(0.8451)$ & $(1.2642$ \\
\hline
\end{tabular}

Note: The first row indicates maturities of the bonds. The first column includes the explanatory variables in the regression. Bus-cycle is a business cycle dummy variable according to NBER business dates. Bus-cycle=1: expansion; Bus-cycle $=0$ : recession. $\mathrm{f} 1=1$-month rate, $\mathrm{f} 6=6$-month forward rate, $\mathrm{f} 60=$ 5 -year forward rate. Numbers in parentheses are Newy-West standard errors. 
Table 3: SNP specifications

\begin{tabular}{ccccccc|c|cc}
\hline$L_{\mu}$ & $L_{r}$ & $L_{p}$ & $K_{z}$ & $I_{z}$ & $K_{x}$ & $I_{x}$ & $l_{\theta}$ & $s_{n}(\hat{\theta})$ & $\mathrm{BIC}$ \\
\hline 1 & 0 & 1 & 0 & 0 & 0 & 0 & 9 & -.82698 & -.76277 \\
2 & 0 & 1 & 0 & 0 & 0 & 0 & 13 & -.85153 & -.75897 \\
3 & 0 & 1 & 0 & 0 & 0 & 0 & 17 & -.85784 & -.73656 \\
\hline 1 & 1 & 1 & 0 & 0 & 0 & 0 & 11 & -1.02082 & -.94234 \\
1 & 2 & 1 & 0 & 0 & 0 & 0 & 13 & -1.05923 & -.96648 \\
1 & 3 & 1 & 0 & 0 & 0 & 0 & 15 & -1.10226 & -.99525 \\
1 & 4 & 1 & 0 & 0 & 0 & 0 & 17 & -1.15356 & -1.03228 \\
1 & 5 & 1 & 0 & 0 & 0 & 0 & 19 & -1.17153 & -1.03598 \\
1 & 6 & 1 & 0 & 0 & 0 & 0 & 21 & -1.17394 & -1.02412 \\
1 & 7 & 1 & 0 & 0 & 0 & 0 & 23 & -1.18247 & -1.01839 \\
\hline 1 & 5 & 1 & 2 & 1 & 0 & 0 & 23 & -1.18124 & -1.01716 \\
1 & 5 & 1 & 2 & 0 & 0 & 0 & 24 & -1.22077 & -1.04956 \\
1 & 5 & 1 & 3 & 2 & 0 & 0 & 25 & -1.18248 & -1.00413 \\
1 & 5 & 1 & 3 & 1 & 0 & 0 & 26 & -1.22941 & -1.04393 \\
1 & 5 & 1 & 3 & 0 & 0 & 0 & 28 & -1.23006 & -1.03030 \\
1 & 5 & 1 & 4 & 3 & 0 & 0 & 27 & -1.22163 & -1.02901 \\
$\mathbf{1}$ & $\mathbf{5}$ & $\mathbf{1}$ & $\mathbf{4}$ & $\mathbf{2}$ & $\mathbf{0}$ & $\mathbf{0}$ & $\mathbf{2 8}$ & $\mathbf{- 1 . 2 5 7 0 5}$ & $\mathbf{- 1 . 0 5 7 2 9}$ \\
1 & 5 & 1 & 4 & 1 & 0 & 0 & 30 & -1.26133 & -1.04703 \\
1 & 5 & 1 & 4 & 0 & 0 & 0 & 33 & -1.28814 & -1.05272 \\
1 & 5 & 1 & 5 & 4 & 0 & 0 & 29 & -1.22625 & -1.01936 \\
1 & 5 & 1 & 5 & 3 & 0 & 0 & 30 & -1.25837 & -1.04435 \\
1 & 5 & 1 & 5 & 2 & 0 & 0 & 32 & -1.26270 & -1.03441 \\
1 & 5 & 1 & 5 & 1 & 0 & 0 & 35 & -1.29353 & -1.04383 \\
1 & 5 & 1 & 5 & 0 & 0 & 0 & 39 & -1.29058 & -1.01235 \\
\hline 1 & 5 & 1 & 4 & 2 & 1 & 0 & 48 & -1.32073 & -.97829 \\
1 & 5 & 1 & 4 & 2 & 2 & 1 & 68 & -1.36002 & -.87490 \\
1 & 5 & 1 & 4 & 2 & 2 & 0 & 78 & -1.37679 & -.82033 \\
\hline & & & & & & & & \\
& & & & & \\
1 & & &
\end{tabular}

Note: $L_{\mu}$ is the number of lags in VAR conditional mean. $L_{r}$ is number of lags in ARCH conditional standard deviation. $K_{z}$ is the degree of the square Hermite polynomial that captures the deviation of the standardized innovation $z$ from conditional Gaussian distribution. The interaction polynomial term above the $I_{z}$ degree is suppressed as zero. The degree of x-polynomial $K_{x}$ is fixed at 0 , and by convention $L_{p}$ is set to be $1 . l_{\theta}$ is the number of coefficients in the SNP model. $s_{n}(\hat{\theta})$ is the negative sample mean log-likelihood. $\mathrm{BIC}$ is the Bayesian Information Criterion. According to BIC, the preferred SNP specification is 10514200. 
Table 4: Parameter Estimates of SNP density

\begin{tabular}{ccc}
\hline Parameter & Estimates & Standard Error \\
\hline $\mathrm{a}(0,0)$ & 1.00000 & 0.00000 \\
$\mathrm{a}(0,1)$ & -0.05179 & 0.06782 \\
$\mathrm{a}(1,0)$ & 0.07111 & 0.06689 \\
$\mathrm{a}(0,2)$ & -0.10675 & 0.05547 \\
$\mathrm{a}(1,1)$ & 0.22368 & 0.06511 \\
$\mathrm{a}(2,0)$ & -0.23111 & 0.08141 \\
$\mathrm{a}(0,3)$ & 0.01787 & 0.01269 \\
$\mathrm{a}(3,0)$ & -0.01448 & 0.01384 \\
$\mathrm{a}(0,4)$ & 0.01470 & 0.00595 \\
$\mathrm{a}(4,0)$ & 0.02222 & 0.00684 \\
\hline$\mu(2,0)$ & -0.00079 & 0.00704 \\
$\mu(1,0)$ & -0.00531 & 0.01195 \\
$\mu(2,2)$ & 0.98033 & 0.01447 \\
$\mu(2,1)$ & 0.00659 & 0.01593 \\
$\mu(1,2)$ & 0.01049 & 0.01401 \\
$\mu(1,1)$ & 0.97699 & 0.01507 \\
$\mathrm{R}(1,0)$ & 0.04068 & 0.00901 \\
$\mathrm{R}(2,0)$ & 0.04830 & 0.00820 \\
$\mathrm{R}(3)$ & 0.08870 & 0.01251 \\
$\mathrm{R}(1,1)$ & 0.33931 & 0.07707 \\
$\mathrm{R}(2,1)$ & 0.18582 & 0.05988 \\
$\mathrm{R}(1,2)$ & 0.22569 & 0.07642 \\
$\mathrm{R}(2,2)$ & 0.11341 & 0.05650 \\
$\mathrm{R}(1,3)$ & 0.16133 & 0.06670 \\
$\mathrm{R}(2,3)$ & 0.25376 & 0.07700 \\
$\mathrm{R}(1,4)$ & 0.12867 & 0.05434 \\
$\mathrm{R}(2,4)$ & 0.03708 & 0.05448 \\
$\mathrm{R}(1,5)$ & 0.09542 & 0.05642 \\
$\mathrm{R}(2,5)$ & 0.00238 & 0.06357 \\
\hline & &
\end{tabular}

This table reports point estimates as well as their standard errors of the parameters in the SNP model (10514200). $a(i, j)$ are parameters of the Hermit polynomial function. $\mu(i, j)$ are parameters of the VAR conditional mean. $R(i, j)$ are parameters of the ARCH standard deviation of the innovation $z$. See Gallant and Tauchen (2001) or Bansal and Zhou (2002) for more detailed interpretations of these parameters. 
Table 5: Parameter Estimates of the Term Structure Models

\begin{tabular}{|c|c|c|c|c|}
\hline & & Model 1 & Model 2 & Model 3 \\
\hline \multirow[t]{10}{*}{ Regime 1} & $a_{0}$ & 0.0058 & 0.0027 & 0.0036 \\
\hline & & $(0.0007)$ & $(0.0002)$ & $(0.0003)$ \\
\hline & $a_{1}$ & -0.0907 & -0.1501 & -0.1488 \\
\hline & & $(0.0096)$ & $(0.0036)$ & $(0.0006)$ \\
\hline & $\sigma$ & 0.0025 & 0.0024 & 0.0025 \\
\hline & & $(0.0002)$ & $(2.6 \mathrm{e}-5)$ & $(0.0003)$ \\
\hline & $\theta_{x}$ & -10.7774 & -15.7689 & -15.5444 \\
\hline & & $(1.7709)$ & $(0.0070)$ & $(0.0053)$ \\
\hline & $\theta_{s}$ & & & 0.1438 \\
\hline & & & & $(0.0337)$ \\
\hline \multirow[t]{10}{*}{ Regime 2} & $\overline{a_{0}}$ & & 0.0086 & 0.0102 \\
\hline & & & $(0.0004)$ & $(0.0004)$ \\
\hline & $a_{1}$ & & -0.0735 & -0.0916 \\
\hline & & & $(0.0022)$ & $(0.0005)$ \\
\hline & $\sigma$ & & 0.0030 & 0.0034 \\
\hline & & & $(1.4 \mathrm{e}-5)$ & $(1.4 \mathrm{e}-5)$ \\
\hline & $\theta_{x}$ & & -17.6468 & -16.9962 \\
\hline & & & $(0.4841)$ & $(0.0975)$ \\
\hline & $\theta_{s}$ & & & -0.0789 \\
\hline & & & & $(0.0109)$ \\
\hline \multirow{7}{*}{$\begin{array}{l}\text { Transition } \\
\text { Intensity }\end{array}$} & $\eta_{s}(1,2)$ & & -1.2040 & -1.1655 \\
\hline & & & $(0.0097)$ & $(0.0263)$ \\
\hline & $\eta_{s}(2,1)$ & & -1.4706 & -1.4457 \\
\hline & & & $(0.0505)$ & $(0.0130)$ \\
\hline & $\chi^{2}$ & 213.34 & 109.70 & 60.38 \\
\hline & Z-value & 27.33 & 15.28 & 7.85 \\
\hline & d.o.f. & 24 & 18 & 16 \\
\hline
\end{tabular}

This table reports EMM estimates of the term structure models. Model 1 refers to the standard 1-factor CIR without regime shifts. Model 2 refers to the 1 -factor CIR model with regime shifts, but the risk of regime shifts is not priced. Model 3 is the 1-factor CIR model developed in Proposition 4 where the risk of regime shifts is priced. $a_{0}, a_{1}$ and $\sigma_{0}$ are the coefficients in the diffusion process of $x(t): d x=\left(a_{0}+a_{1} x\right) d t+\sqrt{\sigma x} d w$. $\theta_{x}$ is coefficient on the market price of diffusion risk, which is given by $\theta_{x} \sqrt{\sigma x}$. $\theta_{s}$ is the coefficient that determines the market price of regime-switching risk $\lambda_{s}(z)$, which is parameterized as $1-e^{\theta_{s}(z)}$ in the model. The transition intensity of the Markov chain is given by $e^{\eta_{s}(z)}$. Numbers in parentheses are the standard errors. The table also reports the $\chi^{2}$ statistics and its degree of freedom (dof) from the EMM estimation. 
Figure 1: Different Regimes of the Yield Curves

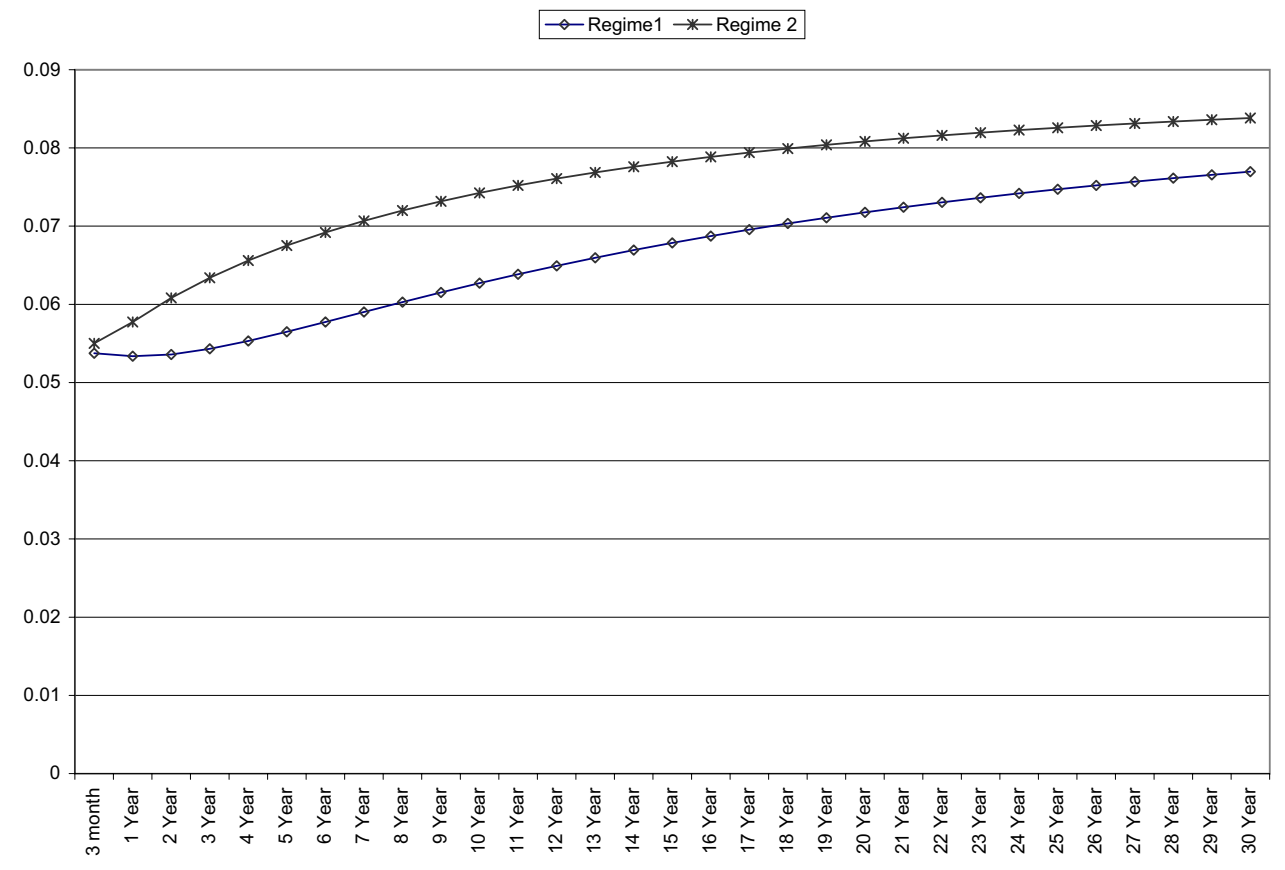

The figure plots the estimated average yield curves (Model 3) in Regime $1(s(t)=1$ and Regime $2(s(2)=2)$ ). When computing the yield curves, we fix the short-term interest rate $r(t)$ at the sample average of the 1month rate of $5.6 \%$. 
Figure 2: Business cycle and the interest rate regimes

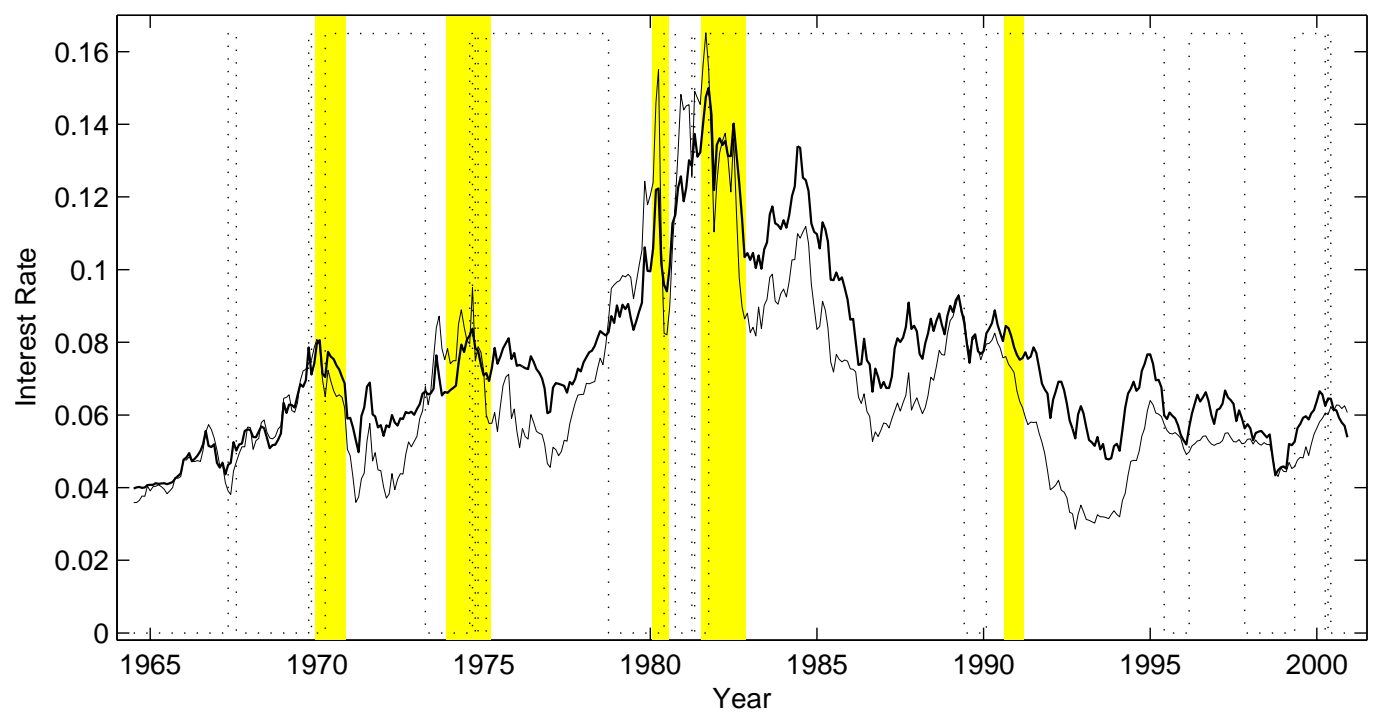

The figure plots the implied regimes by the term structure model (Model 3 in table 5) together with the business cycle. NBER business cycle recessions are indicated the shaded area. The dashed line indicates the regimes implied by the interest rates. We also plot the 6-month (the thinner solid line) and 5-year interest rate (the thicker solid line) in the graph. 
Figure 3: Decompositions of the Term Premiums
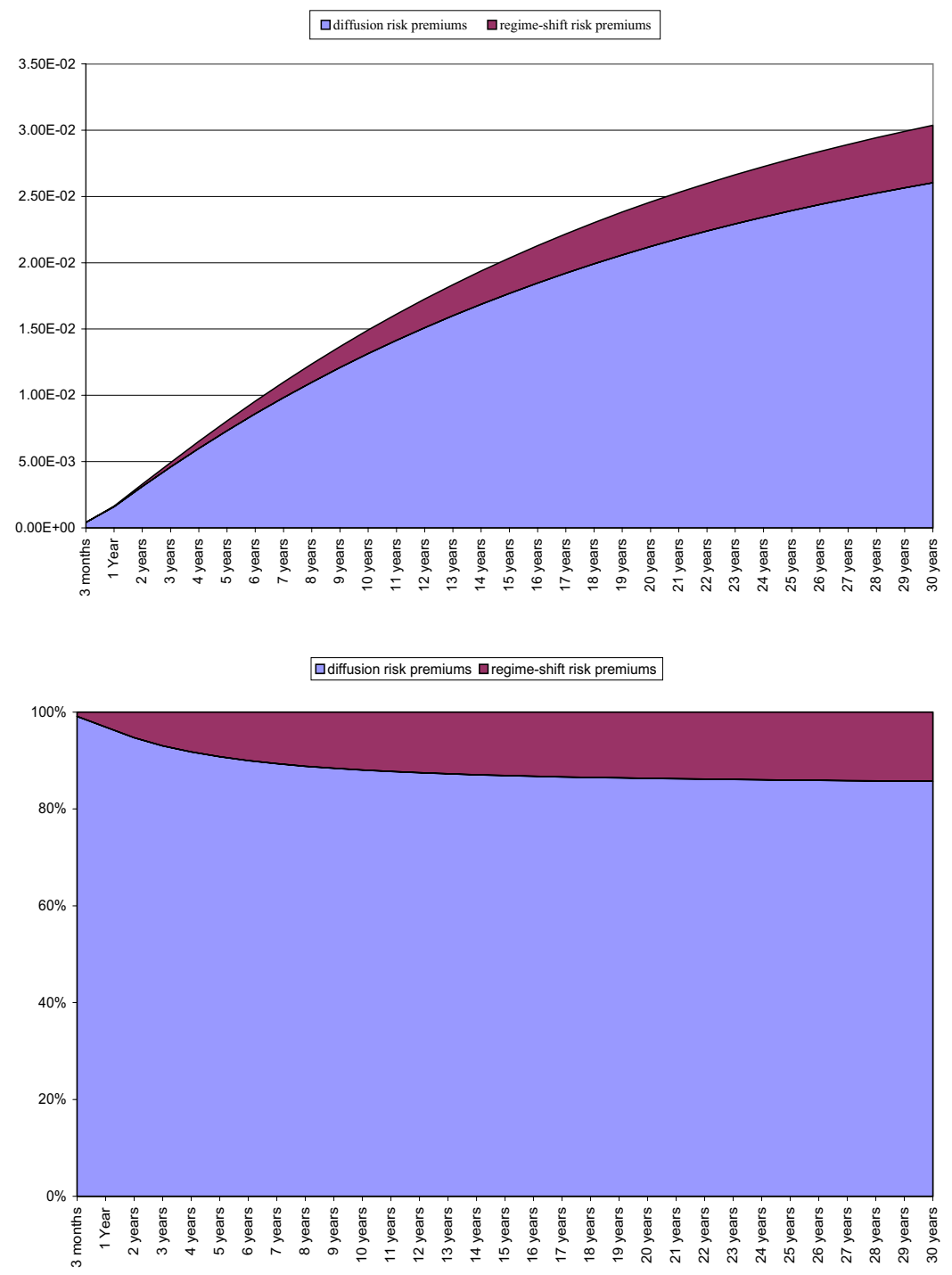

The above two figures report the decompositions of the term premiums for bonds of various maturities (from 3 -month to 30-year). The upper panel plots the bond term premiums as the sum of two components due to the diffusion risk and the regime-shift risk respectively. The lower panel plots these two risk components as percentages of the total term premiums. 
Figure 4: The Impact of Regime-shift Risk on Bond Prices

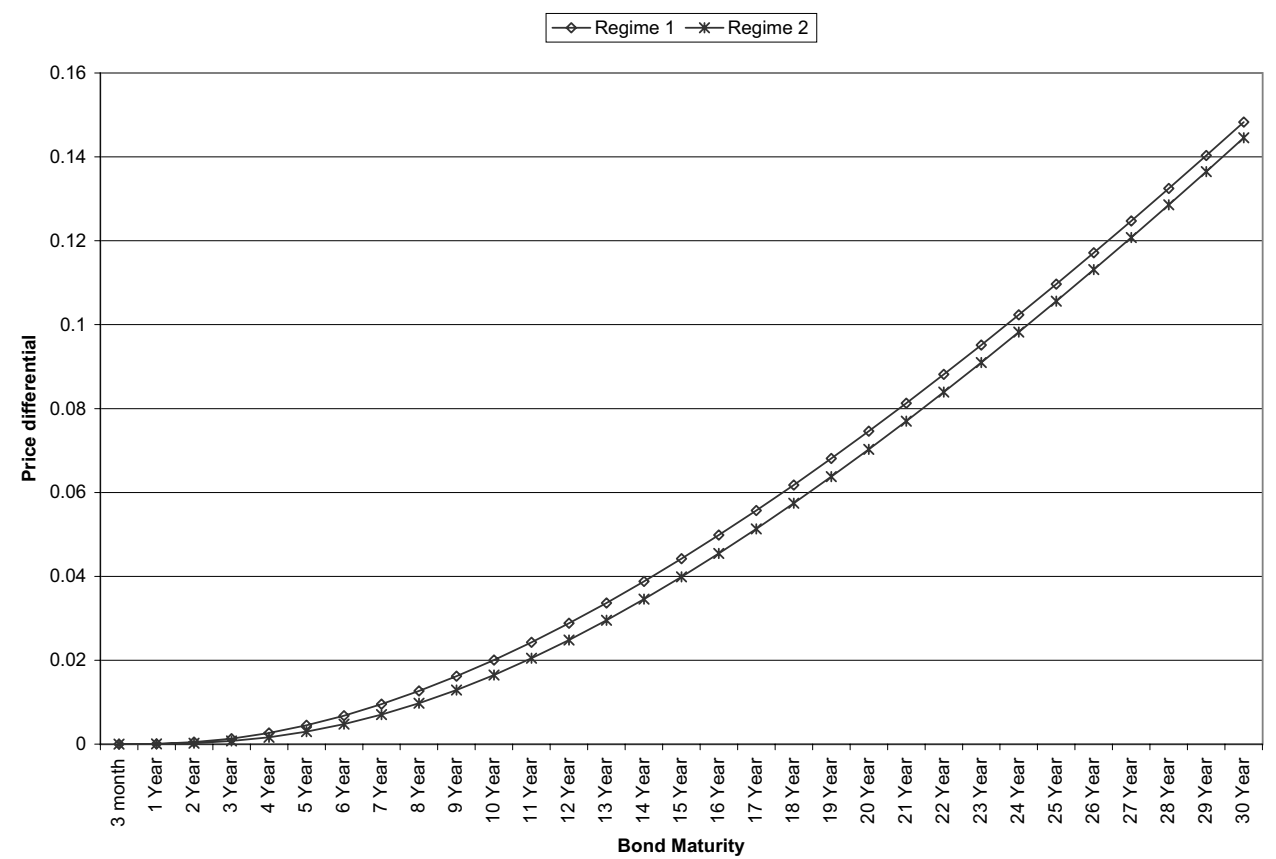

The figure reports the impact of regime-shift risk on bond prices. The price differential is obtained as $\frac{P^{*}(\tau)-P(\tau)}{P(\tau)}$, where $P(\tau)$ is the estimated price of the bond of maturity $\tau$ in Model 3. $P^{*}(\tau)$ is the price of the same bond obtained using the same model and the same parameter values except holding the market price of regime-shift risk at zero $\left(\theta_{s}(z)=0\right)$. In calculating the bond prices, we fix the short-term interest rate $r(t)$ at the sample average of the 1 -month rate of $5.6 \%$. The line with diamonds is the bond price differential in regime 1 . The line with crosses is the bond price differential in regime 2. 
Figure 5: The Impact of Regime-shift Risk on the Yield Curve
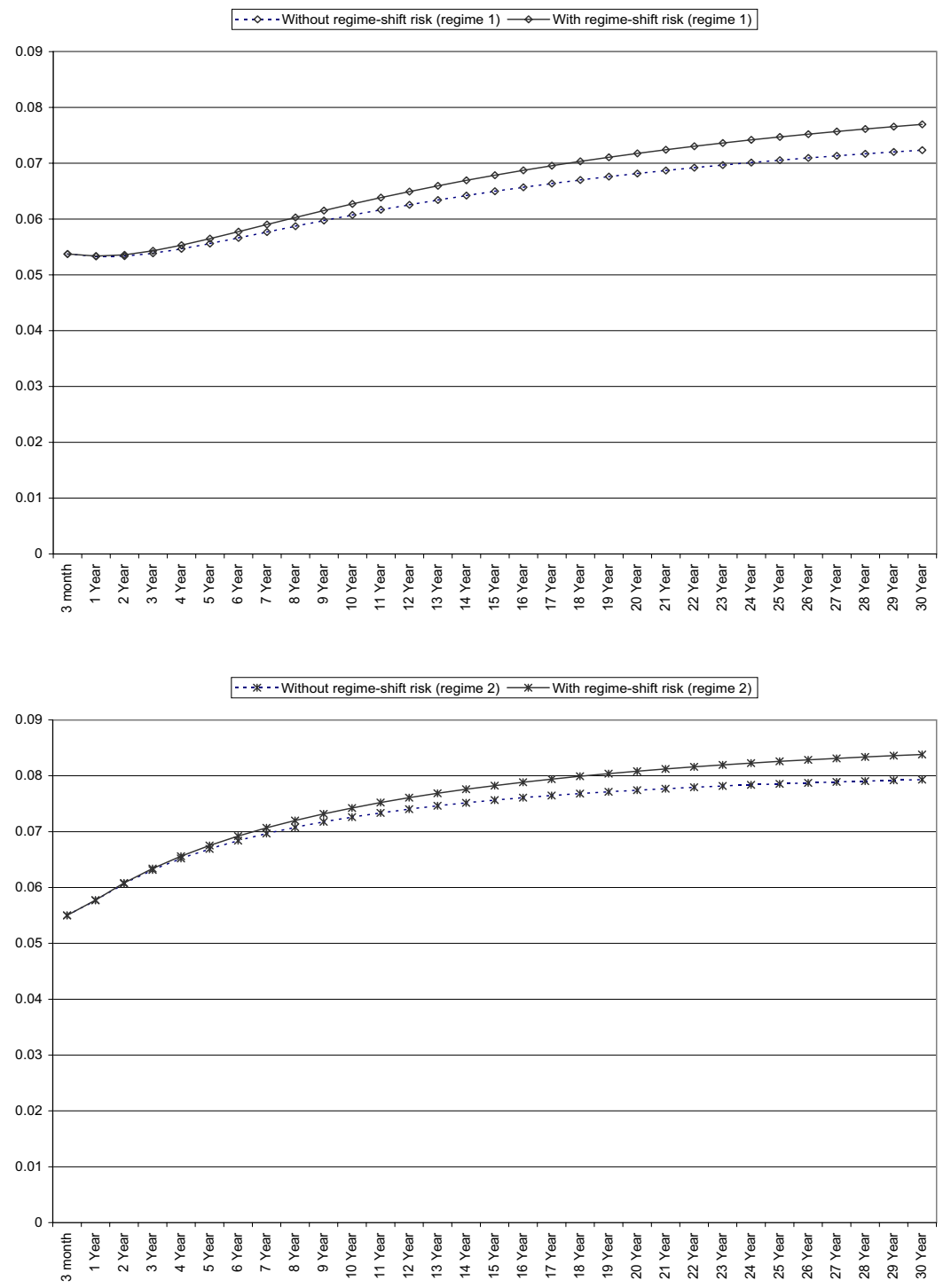

These two figures illustrate the impact of regime-shift risk on the yield curve in Regime 1 (the upper panel) and Regime 2 (the lower panel) respectively. The solid line is the estimated yield curve from Model 3. The dashed line is the yield curve obtained using the same model and the same parameter values except holding the market price of regime-shift risk at zero $\left(\theta_{s}(z)=0\right)$. In calculating the yields, we fix the short-term interest rate $r(t)$ at the sample average of the 1-month rate of $5.6 \%$. 